\title{
Stable superhydrophobic coatings Using PVDF/MWCNT nanocomposite
}

\author{
R. P. S. Chakradhar*, G. Prasad, Parthasarathi Bera, Bharathibai J. Basu, \\ C. Anandan*
}

Surface Engineering Division, CSIR-National Aerospace Laboratories, Bangalore 560017, India.

\begin{abstract}
Thermally stable superhydrophobic coatings have been prepared using Polyvinylidene fluoride (PVDF)-Multiwalled carbon nanotubes (MWCNTs) by spray coating method. The effects of MWCNT $(0-66 \mathrm{wt} \%)$ and temperature (300 to $623 \mathrm{~K})$ on wettability have been studied. A transformation from hydrophobic to superhydrophoic state was achieved with increase of CNT content. X-ray diffraction (XRD) and Fourier transform infrared spectroscopy (FTIR) studies reveal that with increase in CNT content, $\alpha$-phase of PVDF decreases suggesting that MWCNT has strong effect on the phase separation of PVDF. Field emission scanning electron microscopy (FESEM) studies show that the coatings have rough surface with porous structure. With increase in CNT content the protrusion like structures decreased reaching micro/nano scales. The coatings were thermally stable upto $573 \mathrm{~K}$ exhibiting superhydrophobicity and thereafter transformed to superhydrophilic state at $623 \mathrm{~K}$. Energy dispersive X-ray spectroscopy (EDXS) analysis show the absence of fluorine after annealing at $623 \mathrm{~K}$ suggesting decomposition of PVDF. X-ray photoelectron spectroscopy (XPS) of C1s and F1s core levels in as-deposited PVDF-MWCNT coating show the presence of $\mathrm{CF}_{2}$ related species. Concentration of fluorine drastically decreases after heat treatment of the coating at 350 ${ }^{\circ} \mathrm{C}$. The main advantage of the present method is feasibility for application over large area and the coatings are stable upto $573 \mathrm{~K}$.
\end{abstract}

Keywords: Polyvinylidene fluoride; Multiwalled carbon nanotubes; superhydrophobic; superhydrophilic; X-ray diffraction; WCA; FESEM; DTA/TG; FTIR; XPS *Corresponding author: chakra@nal.res.in; canandan@nal.res.in; Tel :+91 080-25026247 


\section{Introduction}

In recent times superhdrophobic surfaces with water contact angle greater than $150^{\circ}$ and hysteresis less than $10^{\circ}$ have attracted considerable attention due to its potential applications in self-cleaning, anti-sticking, anti-icing and anti-corrosion properties [1-7]. Further, the superhydrophobic surface can be applicable in many fields, such as oil-water separation, structural color, fluidic drag reduction, biosurface, battery and fuel cell application, prevention of water corrosion and so on [5]. The approaches used in making a surface superhydrophobic emphasize the importance of a combination of micronanoroughness of the surface and low surface energy. In general, the techniques used involve a two-step procedure in which a surface is first roughened and then modified to lower the surface energy[8-10]. Two models have been proposed to govern this effect. The Wenzel model states that the surface roughness increases the surface area of the solid and thereby enhances the hydrophobicity [11], whereas the Cassie-Baxter model suggests that the air trapped within the grooves beneath the liquid leads to superhydrophobicity as the drop sits partially on the air [12]. In the last decade, several techniques have been developed to fabricate artificial superhydrophobic surfaces, including plasma etching [13], laser etching [14], chemical vapor deposition [15], electrospinning [16], anodic oxidation [17], electrochemical reaction and deposition [18], sol-gel method [19], layerby-layer deposition [20] and so on. However, many artificial methods have certain limitations, like severe conditions, complex process control, special equipment and poor durability. Therefore, inventing some simple and effective methods to fabricate superhydrophobic surfaces with durable performance is an inevitable tendency. 
Carbon nanotubes (CNTs) can be one of candidates to create a surface with a roughness at mirco/nanometer level owing to their rigid cylindrical nanostructures with a diameter ranging from about $1 \mathrm{~nm}$ to several nanometers and length ranging from hundreds of nanometers to micrometers. The research related to superhydrophobic materials based on CNTs has been attractive in the last decade [21-24]. Up to now, superhydrophobic CNTs have been fabricated using functionalized CNTs, such as covalent attachment [25] and noncovalent adsorption or wrapping of various long-chain hydrophobic molecules [26] on the surface of CNTs, which can reduce the surface energy of the CNT surface. A solution method is commonly used to fabricate superhydrophobic materials based on randomly laid CNTs, which is advantageous to subsequent coating. MWCNTs treated with cetyltrimethylammonium bromide and hydroxylic MWCNTs treated with perfluorooctanoic acid in water were found to exhibit a remarkable superhydrophobicity after dried [27,28]. However, limitations exist when polymeric materials are exposed to atmospheric conditions where degradation in superhydrophobic properties occurs due to UV-irradiation and exposure to impurities, $\mathrm{O}_{2}$ and moisture present in the environment.

In the present work we have prepared thermally stable PVDF-MWCNT superhydrophobic coatings by a spray coating method. PVDF, a semi-crystalline polymer exists in four different forms. The existence of different phases in PVDF depends on synthesis conditions like solvent, melt temperature, method of casting, stretching of thin films and annealing conditions [29]. The prepared coatings were well characterized by using X-ray diffraction (XRD), Field emission scanning electron microscopy (FESEM), Fourier transform infrared spectroscopy (FTIR), Energy dispersive X-ray spectroscopy, 
3D surface profiler and X-ray photoelectron spectroscopy (XPS). The thermal stability of the coatings was also evaluated for their possible usage in high temperature applications.

\section{Experimental}

Polyvinylidene fluoride (PVDF) was procured from M/s Pragathi Chemicals, India and multiwalled carbon nanotubes MWCNT (diameter ranging from 4 to $15 \mathrm{~nm}$ and length ranging from 30 to $15 \mu \mathrm{m}$ ) was procured from Intelligent Materials Pvt. Ltd, India. The solvents, acetone and $\mathrm{N}, \mathrm{N}$ dimethyl formamide (DMF), were obtained from Merck, India. Water was purified by a Millipore water purifying system. $100 \mathrm{mg}$ of asreceived PVDF was mixed in $10 \mathrm{ml}$ DMF and magnetically stirred for 10 min to get transparent solution. The solution was then transferred into a spray gun and coated on

glass and $\mathrm{Al}$ substrates and dried at room temperature overnight. The thickness of the coatings was $\sim 10 \mu \mathrm{m}$.

A mixture of PVDF and different percentages of MWCNT $(10,20,25,33,50$ and 66 wt.\%) were dispersed in $10 \mathrm{ml}$ of DMF using a magnetic stirrer (10 min) and then ultrasonicated for $50 \mathrm{~min}$. The mixture was poured into a beaker that contains 80 $\mathrm{ml}$ of milliQ water under stirring; a black precipitate was formed. The precipitate was collected using Whatman filter paper and washed several times with milliQ water and dried at room temperature and kept overnight in a vacuum oven at $70{ }^{\circ} \mathrm{C}$.

The superhydrophobic coatings were prepared by using the as-prepared precipitated PVDF-MWCNT composite dissolved in acetone and subjected to ultrasonication for 
about $30 \mathrm{~min}$. The sonicated mixture was transferred in to a spray gun and then sprayed on glass and aluminum substrates and cured at room temperature for $8 \mathrm{~h}$.

The phase purity of the coatings was examined by XRD (Bruker D8 Advace) by using $\mathrm{Cu}$ $\mathrm{K}_{\alpha}$ radiation with a nickel filter. The surface morphology of the coatings was examined using FESEM (model Carl Zeiss Supra 40). The 3D roughness profile was measured using 3D profilometer (model Nano Map 500LS from AEP Technology, USA). FTIR studies were performed on a Shimadzu IRAffinity-1 spectrometer with $\mathrm{KBr}$ pellets. The water contact angle (WCA) of the coatings was measured using a contact angle analyzer (model Phoenix 300 Plus from M/s Surface Electro Optics, South Korea). Measurements were made using tangent linefitting mode. Water sliding angle (SA) was measured using a home-made instrument. The drop volume for WCA and SA measurements was $8 \mu \mathrm{L}$. Deionised milli Q water was used for measurements. An average of five measurements was taken for reporting WCA and the error in measurements is within $\pm 2^{\circ}$. The thickness of the coatings was measured by Mitutoyo digital micrometer and was found to be in the range 10-12 $\mu \mathrm{m}$. XPS of as-prepared and heat treated PVDF-CNT samples were recorded with a SPECS spectrometer using $150 \mathrm{~W}$ nonmonochromatic $\mathrm{AlK} \alpha$ radiation $(1486.6 \mathrm{eV})$ as an $\mathrm{X}$-ray source.. The binding energies reported here were referenced with $\mathrm{C} 1 \mathrm{~s}$ peak at $284.6 \mathrm{eV}$ with a precision of $\pm 0.1 \mathrm{eV}$. All the spectra were obtained with pass energy of $25 \mathrm{eV}$ and step increment of $0.05 \mathrm{eV}$. The experimental data were curve fitted into several components with GaussianLorentzian peaks after Shirley background subtraction employing CasaXPS program. The spin-orbit splitting and doublet intensities were fixed as given in the literature [30]. 


\section{Results and discussions}

\subsection{X-ray diffraction studies}

Fig. 1 shows the XRD pattern of (a) as-received PVDF and (b) phase separated PVDF. PVDF exhibits at least four crystalline forms, i.e. $\alpha-, \beta-, \gamma-$, and $\delta$-phases. Among the four polymorphs, the $\alpha$-phase is the most common and stable polymorph, while the $\beta$ phase is the most important one for several applications like (piezoelectric and pyroelectric properties and exhibits outstanding performance when used in practical devices). The $\beta$-phase may be obtained by drawing films containing $\alpha$-phase [31], by applying an extremely high electric field to the $\alpha$-phase of PVDF [32], by crystallization from solution under special conditions [33], or by crystallization from the melt [34]. In Fig. 1(a) diffraction peaks at $2 \theta$ values of $18.3^{\circ}, 20^{\circ}$, and $26^{\circ}$ are due to the (100), (110), and (021) reflections of $\alpha$-phase of PVDF and the peak at $2 \theta=39^{0}$ corresponds to $\gamma$ phase of PVDF and is assign to (211) reflection [35]. In Fig. 1(b), for the phase separated PVDF, it is interesting to observe that upon phase separation the diffraction peaks at $2 \theta$ values $18.3^{\circ}$ and $26^{\circ}$ almost disappears suggesting a reduction in $\alpha$-phase. Further, a new peak at $2 \theta$ value of $20.2^{\circ}$ that corresponds to $\beta$ phase appears [36,37]. The peak at $2 \theta=39^{\circ}$ remains

Fig. 2 shows the X-ray diffraction pattern of PVDF-66 wt.\% MWCNT superhydrophobic coatings at (a) room temperature $(300 \mathrm{~K})$, (b) calcined at $623 \mathrm{~K}(1 \mathrm{~h})$ and (c) pure MWCNT respectively. From Fig. 2(a) we can observe that with incorporation of MWCNT to PVDF, a diffraction peak at $2 \theta$ value of $26.2^{\circ}$ is formed in addition to $\beta$-phase of PVDF. Levi et al. [38] observed that the addition of MWCNTs led 
to $\beta$-phase formation in PVDF by sonication. We have observed that with addition of MWCNT, the diffraction peak intensities of $\alpha$-phase of PVDF at $2 \theta$ values $18.3^{\circ}$ and $20.02^{\circ}$ diminish which might be due to phase separation. The new peak at $2 \theta=26.2^{\circ}$ can be assigned to the MWCNT $[39,40]$. Upon calcination at $623 \mathrm{~K}$ the $\beta$-phase of PVDF disappears leaving the MWCNT peak intact at $2 \theta=26.2^{\circ}$. That the disappearance of $\beta$ phase might be due to the decomposition of PVDF after $613 \mathrm{~K}$, was supported by our TGA results which will be discussed in following sections.

\subsection{Wettability studies}

Fig. 3 shows the water contact angle (WCA) images of PVDF-MWCNT with different MWCNT concentrations: (a) $0 \%$, (b) 10\%, (c) 20\%,(d) 25\%, (e) 33\%, (f) 50\%, (g) $66 \%$ respectively. From Fig. 3(a) we can observe that the as received PVDF coatings exhibit WCA of $\approx 105^{\circ}$ which is hydrophobic in nature. Upon phase separation the WCA slightly increases to $112^{\circ}$. As we can see from Fig. 3 the superhydrophobicity is achieved by introducing MWCNT. By increasing MWCNT content the WCA increases from $105^{\circ}$ to $154^{\circ}$ and the sliding angle decreases correspondingly and these values are tabulated in Table 1.

\subsubsection{Effect of temperature (300-623 K) on wettability}

The thermal stability of the PVDF-MWCNT superhydrophobic coatings was studied by annealing the coatings at different temperatures $(373,423,473,523,573,623$ $\mathrm{K}$ for $1 \mathrm{~h}$ ) and measuring WCA after each treatment at room temperature. Fig. 4 shows the variation of WCA of different weight percent MWCNT/PVDF superhydrophobic 
coatings as a function of temperature. It reveals that the WCA for the $10-25 \%$, MWCNT containing films gradually decreases with temperature and reaches almost $10^{\circ}$ at $623 \mathrm{~K}$. In case of 33-66 wt. $\%$ MWCNT containing films the WCA $\left(>150^{\circ}\right)$ is almost constant upto $573 \mathrm{~K}$. The water contact angle, sliding angle of the coatings heat teated at different temperatures are listed in Table 2. Fig. 5 shows the water droplets on the PVDF/MWCNT superhydrophobic coatings in (a) as-prepared condition and after heat treatment at (b) 573 $\mathrm{K}$ and (c) $623 \mathrm{~K}$. It is interesting to observe that at $623 \mathrm{~K}$ a transformation from superhydrophoic to super hydrophilic state is achieved reaching $\mathrm{WCA}<10^{\circ}$. The unusual wetting characteristics are governed by both chemical composition and the geometric microstructure of the surface. These studies suggest that the coatings are thermally stable upto $573 \mathrm{~K}$ exhibiting superhydrophobicity and thereafter they transform to superhydrophilic state at $623 \mathrm{~K}$.

Shansheng Yu et al [41] have prepared PVDF-MWCNT composite by sonication method. In their study they report that $\alpha$-phase co-exists with $\beta$-phase in the composite. Further, it has been reported that there exist a very large energy barrier between transgauche-trans-gauche (TGTG) and trans-trans (TT) conformations of PVDF molecule. In addition, they all can be absorbed easily on the CNT surface and act as nucleating agents. A possible mechanism was proposed by them for $\beta$-phase formation in the PVDF-MWCNT composite prepared by sonication. The authors found that the TT molecular chain prefers to be absorbed on the CNT surface compared with TGTG molecular chain, and the configuration in which $\mathrm{H}$ atoms and CNT surface are face-toface are more stable than that where F atoms and CNT surface are face-to-face [41]. The electro negativity value of $\mathrm{C}$ atom is between $\mathrm{H}$ and $\mathrm{F}$. Hence, in PVDF, the negative 
charge transfer takes place from $\mathrm{H}$ to $\mathrm{C}$ atom and the negative charge is accumulated around the $\mathrm{F}$ atoms. The interaction between the $\mathrm{H}$ atom with positive charge in PVDF and $\mathrm{C}$ atom with $\pi$ orbital in $\mathrm{CNT}$ is stronger than that between the $\mathrm{F}$ atom with negative charge and $\mathrm{C}$ atom with $\pi$ orbital. In addition, they have observed that the different adsorption energies between zigzag and armchair CNTs are very small. Based on the above facts we can assume that in PVDF-MWCNT composite the $-\mathrm{CF}_{2}-$ may be projected on the outward/away from MWCNT axis which might also be responsible for higher water repellence. The schematic of such a representation is shown in Fig. 6.

\subsection{Surface microstructure and surface roughness of the coatings}

Wettability is affected by surface microstructure, roughness and surface free energy. We have observed different wettability characteristics for different weight percentage of MWCNTS in PVDF matrix. In order to understand this phenomenon surface morphology of the coatings was examined by using FESEM. The WCA of $10 \%$ PVDF-MWCNT shows $122^{\circ}$ with a SA of $>90^{\circ}$ and the corresponding FESEM images with different magnifications are shown in Fig.7. As we can see clearly from Fig. 7 at lower MWCNT concentrations the coatings have more porous structure. Fig. 8 shows FESEM images of PVDF composite coatings with 33\%MWCNT at different magnifications. With increase of MWCNT to $33 \%$ the porosity slightly decreases with creation of protrusion like structures which can be clearly seen at higher magnifications thereby increasing the WCA to $150^{\circ}$ with $\mathrm{SA} \sim 10^{\circ}$.

In general, the hydrophilic and hydrophobic coatings have lower average roughness $R_{a}$ and rms roughness $\left(R_{q}\right)$ whereas the roughness was high for SH coatings. 
Increase of MWCNT to $66 \%$, leads to enhanced surface roughness with creation of more protrusion like structures (Fig. 9) and the wettability of the surface changes according to Cassie-Baxter's law increasing WCA to $154^{\circ}$ with SA $<3^{\circ}$. The higher WCA and lower SA at 66\% MWCNT makes these coatings superhydrophobic due to the creation of micro/nano scale roughness

The 3D surface topography of $66 \%$ MWCNT-PVDF superhydrophobic coating is shown in Fig. 10. The average roughness $\left(\mathrm{R}_{\mathrm{a}}\right)$ and $\mathrm{Rq}$ of such coatings were found to be 2.903 micron and 3.647 micron respectively. Further, it has been observed that upto $573 \mathrm{~K}$ all the coatings exhibit superhydrophobicity and the morphology shows the MWCNTs covered with polymer. It is interesting to observe that at $623 \mathrm{~K}$ a transformation from superhydrophoic to super hydrophilic state is achieved reaching $\mathrm{WCA}<10^{\circ}$.

Fig. 11 shows FESEM images PVDF-66\%MWCNT coatings calcined at $623 \mathrm{~K}$ at different magnifications. From these micrographs we can see that upon calcination, the polymer decomposition takes place leaving the CNTs alone. The composition of the coatings has been evaluated by energy dispersive X-ray spectroscopy (EDXS) and is shown in Fig.12. The EDXS analysis shows the absence of fluorine after heating at $623 \mathrm{~K}$ suggesting decomposition of PVDF. In order to confirm the decomposition of the polymer we have performed TGA-DTA studies which will be discussed in sub sequent sections. Hence it can be concluded that the changes in the morphology and loss of fluorine after heating PVDF above its decomposition temperature has resulted in loss of superhydrophobicity. 


\subsection{TGA-DTA studies}

TGA measurement was carried out to find the detailed changes in PVDF-MWCNT composite coatings. Fig. 13 (A) shows the DTA curves and Fig. 13 (B) shows the corresponding TGA curves of PVDF-MWCNT with different MWCNT concentrations: (a) $33 \%$ (b) $66 \%$ and (c) $66 \%$ calcined at $623 \mathrm{~K}$ respectively.

From Fig. 13 (A) we can clearly see that for 33\% MWCNT- PVDF coatings, the exothermic peak (around $633 \mathrm{~K}$ ) of DTA indicates that the combustion of matrix begins and it peaks around $415 \mathrm{~K}$. The corresponding TGA curve also confirms that weigh loss is initiated at around $633 \mathrm{~K}$ (Fig. 13. B(a)). With the addition of higher MWCNT (66\%) the onset of combustion shifted to $631 \mathrm{~K}$. This indicates that lesser matrix content started to disintegrate at slightly lower temperature. However, this difference is not visible in TGA (Fig. $13 \mathrm{~B}(\mathrm{~b})$ ). Further, in calcined sample the combustion peak did not appear indicates that combustion is completed during the calcination itself. In all the cases the decomposition takes place in two stages. The first one corresponds to the decomposition of polymer and the second one corresponds to decomposition of CNTs. In addition to DTA, TGA curve indicates that there is a gradual degradation between $473-623 \mathrm{~K}$ about $10 \%$ of the weight loss occurring in this range of temperature.

\subsection{FTIR studies}

Fig. 14 shows the FTIR spectra for PVDF-MWCNT with different MWCNT concentrations: (a) $0 \%$, (b) $10 \%$, (c) $33 \%$, (d) $66 \%$, and (e) $66 \%$ calcined at $623 \mathrm{~K}$, respectively. The IR spectra show characteristics bands at 488, 532, 613, 764, 796, 974, 
1213 , and $1402 \mathrm{~cm}^{-1}$ which can be assigned to $\alpha$-phase. The bands at 508, 841, and 1278 $\mathrm{cm}^{-1}$ are attributed $\beta$-phase [42]. It is observed that for the composites prepared by sonication followed by phase separation the $\alpha$-phase peaks vanish. However, the band at $840 \mathrm{~cm}^{-1}$ of $\beta$-phase corresponding to the $-\mathrm{CF}_{2}$ stretching remains. After calcinations at

$623 \mathrm{~K}$ for 1 hour the peak $840 \mathrm{~cm}^{-1}$ disappear suggesting decomposition of PVDF. The energy dispersive X-ray spectroscopy analysis also confirms the absence of fluorine at $623 \mathrm{~K}$. Further, TGA results also suggest that a clear decomposition of the polymer after $610 \mathrm{~K}$ which is in consistent with the observed results.

\subsection{XPS studies}

XPS studies have been carried out to get information about the composition of asprepared and heat treated PVDF-66\% MWCNT samples. Survey scan from pure PVDF, 66\% MWCNT doped PVDF coating before and after heating at $613 \mathrm{~K}$ and pure MWCNT are shown in Fig. 15. The survey scan shows the presence of $F$ in undoped and asprepared MWCNT doped PVDF film. After heating the doped film to $613 \mathrm{~K}$, fluorine is more or less absent from the coating. Relative surface concentration ratios (atomic \%) of F/C in the coating were 0.29 and 0.01 , respectively before and after heat treatment of PVDF- $66 \%$ MWCNT sample. Therefore, concentration of $\mathrm{F}$ in the sample has been found to decrease drastically after heat treatment due to decomposition of PVDF.

XPS of C1s core levels in as-prepared and heat treated PVDF-66\% MWCNT are shown in Fig. 16. C1s core level of pure MWCNT is also given for comparison. In Fig16 (a) high resolution spectrum from $\mathrm{C} 1 \mathrm{~s}$ core level of as-prepared PVDF-66\% MWCNT sample is broad and asymmetrical indicating the presence of several carbon 
components in the sample. Two dominant peaks one centered at $284.6 \mathrm{eV}$ and another at $290.3 \mathrm{eV}$ can be seen in the spectrum. The peak at $290.3 \mathrm{eV}$ corresponds to carbon bonded as in $-\mathrm{CF}_{2}-$. In the spectrum from the heat treated sample, the peak at $290.3 \mathrm{eV}$ is absent and the broadness of spectral envelope also decreases. Carbon bonded to oxygen species in 286-289 eV binding energy range might have come from the solvent during preparation. Intensity of peak related to $\mathrm{CF}_{2}$ decrease significantly after heat treatment at $350^{\circ} \mathrm{C}$ (Fig. 16 (b)). Comparison of C1s core level spectrum of pure CNT in Fig 16 (c) with that of heat treated sample in Fig 16 (b) shows that coating mainly consists of MWCNT after heating.

F1s core level spectra of as-prepared and heat treated PVDF-66\% MWCNT samples are displayed in Fig. 17. F1s spectrum of as-prepared PVDF-66\% MWCNT sample shows a symmetrical peak at $687.2 \mathrm{eV}$ that is attributed to fluorine moieties with covalent $\mathrm{C}-\mathrm{F}$ bonds in PVDF [43]. Intensity of F1s core level peak drastically decreases when sample is heat treated at $350^{\circ} \mathrm{C}$.

\section{Conclusions}

Polyvinylidene fluoride, PVDF-MWCNT superhydrophobic coatings have been prepared by a facile, phase separation and spray coating method. A transformation from hydrophobic to superhydrophoic state was achieved with increase of MWCNT content. It was observed that $33 \%$ MWCNT is the optimum concentration required to achieve superhydrophobicity. XRD and FTIR studies show that with increase in MWCNT content, $\alpha$-phase in PVDF decreases suggesting that MWCNT has strong effect on the phase formation. The coatings retain their superhydrophobicity on heating upto up to 573 K. Upon heating at $623 \mathrm{~K}$, PVDF in the PVDF-MWCNT coating decomposes with the 
loss of fluorine from the coating, as evidenced from EDS and XPS and a transformation from superhydrophoic to super hydrophilic state is achieved.

Acknowledgments: The authors are grateful to Mr. Shyam Chetty, Director, NAL Bangalore, for permission to publish the work. We thank Mr. Siju and Mr. V. Praveen Kumar for FESEM and 3D roughness measurements. 


\section{References}

[1] R. Blossey, Nature Mater. 2 (2003) 301-306.

[2] L. Mishchenko, B. Hatton, V. Bahadur, J.A. Taylor, T. Krupenkin, ACS Nano. 4 (2010) 7699-7707.

[3] F.C. Wang, C.R. Li, Y.Z. Lv, F.C. Lv, Y.F. Du, Cold Reg. Sci. Technol. 62 (2010) $29-33$.

[4] F.Z. Zhang, L.L. Zhao, H.Y. Chen, S.L. Xu, D.G. Evans, X. Duan, Angew. Chem. Int. Ed. 47 (2008) 2466-2469.

[5] H. Cho, D. Kim, C. Lee, W. Hwang, Current Appl. Phys., 13 (2013) 762-767.

[6] C.P Hsu, L.Y. Chang, C. W. Chiu, P.T.C. Lee, J.J. Lin, ACS Appl. Mater. \& Interf. 5 (2012) 538-545.

[7] J. Hasan, H.K. Webb, V.K. Truong, G.S. Watson, J.A. Watson, M.J. Tobin, G. Gervinskas, S. Juodkazis, J.Y. Wang, R.J. Crawford, E.P. Ivanova, Langmuir 28 (2012) 17404-17409.

[8] X. Zhang, F. Shi, J. Niu, Y. Jiang, Z. Wang, J. Mater. Chem. 18 (2008) 621- 633.

[9] Zhi-Guang Guo, Wei-Min Liu, Bao-Lian Su, Appl. Phys. Lett. 92 (2008) 063104.

[10] K. Liu, M. Zhang, J. Zhai, J. Wang, L. Jiang, Appl. Phys. Lett. 92 (2008) 183103183106.

[11] R. N. Wenzel, Ind. Eng. Chem., 28 (1936) 988-994.

[12] A.B.D. Cassie, S. Baxter, Trans. Faraday Soc., 40 (1944) 546- 551

[13] K. Teshima, H. Sugimura, Y. Inoue, O. Takai, A. Takano, Appl. Surf.Sci. 244 (2005) 619-622.

[14] X.Y. Song, J. Zhai, Y.L. Wang, L. Jiang, J. Phys. Chem. B 109 (2005) 4048-4052. 
[15] L. Huang, S.P. Lau, H.Y. Yang, E.S.P. Leong, S.F. Yu, S. Prawer, J. Phys. Chem. B 109 (2005) 7746-7748.

[16] M. Ma, R.M. Hill, J.L. Lowery, S.V. Fridrikh, G.C. Rutledge, Langmuir 21 (2005) $5549-5554$.

[17] M. Thieme, R. Frenzel, S. Schmidt, F. Simon, A. Hennig, H. Worch, K. Lunkwitz, D. Scharnweber, Adv. Eng. Mater. 3 (2001) 691-695.

[18] X. Zhang, F. Shi, X. Yu, H. Liu, Y. Fu, Z.Q. Wang, J. Am. Chem. Soc.126 (2004) 3064-3065.

[19] X.J. Feng, L. Feng, M.H. Jin, J. Zhai, L. Jiang, D.B. Zhu, J. Am.Chem. Soc. 126 (2004) 62-63.

[20] F. Shi, Z.Q. Wang, X. Zhang, Adv. Mater. 17 (2005) 1005-1009.

[21] L.B. Zhu, Y.H. Xiu, J.W .Xu, P.A. Tamirisa, D.W. Hess, and C.P. Wong, Langmuir 21 (2005) 11208-11212.

[22] T. Sun, G. Wang, H. Liu, L. Feng, L. Jiang, and D. Zhu, J. Am. Chem. Soc., 125 (2003) 14996-14997.

[23] S. Li, H. Li, X. Wang, Y. Song, Y. Liu, L. Jiang, et al., J. Phys. Chem. B, 106 (2002) 9274-9276.

[24] T. Wu, Y. Pan, E. Liu, L. Li, Colloid. Surf. A, 384 (2011) 47-52

[25] V. Georgakilas, A.B. Bourlinos, R. Zboril, C. Trapalis, Chem. Mater. 20 (2008) $2884-2886$.

[26] S. Srinivasan, V.K. Praveen, R. Philip, A. Ajayaghosh, Angew. Chem. Int. Ed. 47 (2008) 5750-5754. 
[27] D. Xu, H. Liu, L. Yang, Z. Wang, Carbon 44 (2006) 3226-3231.

[28] X.H. Men, Z.Z. Zhang, H.J. Song, K. Wang, W. Jiang, Appl. Surf. Sci. 254 (2008) $2563-2568$.

[29] M. G. Buonomenna, P. Macchi, M. Davoli, E.Drioli, Euro. Polym. J.43 (2007) 1557.

[30]. D. Briggs, M.P. Seah, Practical Surface Analysis by Auger and X-ray Photoelectron Spectroscopy, Wiley, New York, 1984.

[31] L. David, J.I. Winsor, B.A. Scheinbeim, J. Polym. Sci., Part B 34 (1996) 2967 2977.

[32] J. Scheinbeim,C. Nakafuku, B.A. Newman, K.D. Pae, J. Appl. Phys., 50 (1979) 4399.

[33] R.L. Miller, J. Raisoni, J. Polym. Sci., Phys. Ed., 14 (1976) 2325 - 2326.

[34] A.J. Lovinger, Polymer, 22 (1981) 412 - 415.

[35] D. M. Estterly, B. J. Love, J. Polymer Sci., B : Polym. Phys. 42 (2004) 91-97.

[36] M. El Achaby, F.Z. Arrakhiz, S. Vaudreuil, E.M. Essassi, A. Qaiss, Appl. Surf. Sci., 258 (2012) $7668-7677$

[37] M.El Achaby, F.Z. Arrakhiz, S. Vaudreuil, E. Essassi, A. Qaiss, M. Bousmina, Polym. Eng. Sci., 53 (2013) 34-43

[38] N. Levi, R. Czerw, S.Y. Xing, P. Lyer, D. L.Carroll, Nano Lett., 4 (2004) 1267.

[39] D. Thiele, E. Lopez-Camacho Colmenarejo, B. Grobety, A. Züttel,Diamond and Related Materials 18 (2009) 34 - 38 
[40] M.A. Bavio and A.G. Lista, Scientific World Journal. 2013 (2013): 387458

[41] S. Yu,W. Zheng, W. Yu,Y. Zhang, Q. Jiang, Z. Zhao, 42 Macromolecules (2009) 8870-8874.

[42] G. H. Kim, S. M. Hong, Y. Seo, Phys. Chem. Chem. Phys. 11 (2009) 10506- 10512

[43]. A. Tressaud, E. Durand, C. Labrugère, J. Flour. Chem. 125 (2004) 1639 - 1648. 


\section{Table Captions}

Table 1 : Composition, Water contact angle and sliding angle of the studied coatings at room temperature

Table 2 : Water contact angle, sliding angle of the studied coatings at different temperatures 


\section{Figure Captions}

Fig. 1. X-ray diffraction pattern of (a) as-received PVDF and (b) phase separated PVDF. Fig. 2. X-ray diffraction pattern of PVDF-66 wt.\% MWCNT superhydrophobic coatings at (a) room temperature $(300 \mathrm{~K})$, (b) calcined at $623 \mathrm{~K}(1 \mathrm{~h})$ and (c) pure MWCNT Fig. 3. Images of water drops on PVDF-MWCNT with different MWCNT concentrations: (a) $0 \%$, (b) $10 \%$, (c) $20 \%$, (d) 25\%, (e) $33.33 \%$, (f) $50 \%$, (g) $66.66 \%$. Fig. 4. Variation of WCA with different annealing temperature in PVDF/66\%MWCNT coatings

Fig. 5. Water droplets on the PVDF-MWCNT superhydrophobic coatings (a) $300 \mathrm{~K}$ and after heat treatment at (b) $573 \mathrm{~K}$ and (c) $623 \mathrm{~K}$

Fig. 6. Schematic representation of $-\mathrm{CF}_{2}-$ projection on the outer surface of MWCNT Fig. 7. FESM images of PVDF-MWCNT 10\% at different magnifications (a) 5KX, (b) $25 \mathrm{KX}$, (c) $50 \mathrm{KX}$, (d) $100 \mathrm{KX}$

Fig. 8. FESM images of PVDF-MWCNT 33\% at different magnifications (a) 5KX, (b) $25 \mathrm{KX}$, (c) $50 \mathrm{KX}$, (d) $100 \mathrm{KX}$

Fig. 9. FESM images of PVDF-MWCNT 66\% at RT for different magnifications (a) $5 \mathrm{KX}$, (b) $25 \mathrm{KX}$, (c) $50 \mathrm{KX}$, (d) $100 \mathrm{KX}$

Fig. 10. 3D surface roughness of 66\% MWCNT-PVDF superhydrophobic coating Fig. 11. FESM images of PVDF-MWCNT 66\%@623 K at different magnifications (a) $5 \mathrm{KX}$, (b) $25 \mathrm{KX}$, (c) $50 \mathrm{KX}$, (d) $100 \mathrm{KX}$ 
Fig. 12. Energy dispersive X-ray spectra of (a) $33 \%$ (b) $66 \%$ and (c) $66 \%$ calcined at 623K PVDF-MWCNT coatings

Fig. 13. (A) DTA curves and (B) corresponding TGA curves of PVDF-MWCNT with different MWCNT concentrations: (a) 33\%, (b) 66\%, and (c) $66 \%$ calcined at $623 \mathrm{~K}$ Fig. 14. FTIR spectra for PVDF-MWCNT with different MWCNT concentrations: (a) $0 \%$, (b) $10 \%$, (c) $33 \%$, (d) $66 \%$, and (e) $66 \%$ claimed at $623 \mathrm{~K}$

Fig. 15. Survey spectra of (a) pure PVDF, (b) PVDF-66\% MWCNT, (c) PVDF-66\% MWCNT after heated at $350^{\circ} \mathrm{C}$ and (d) pure MWCNT.

Fig. 16. C1s core level spectra of (a) as-prepared PVDF-66\% MWCNT and (b) PVDF-66\% MWCNT heat treated at $350{ }^{\circ} \mathrm{C}$ and (c) pure MWCNT.

Fig. 17. F1s core level spectra of (a) as-prepared PVDF-66\% MWCNT and (b) PVDF- $66 \%$ MWCNT heat treated at $350{ }^{\circ} \mathrm{C}$. 


\section{Table 1}

\begin{tabular}{cccccc}
\hline S.No & $\begin{array}{c}\text { PVDF: } \\
\text { MWCNT }\end{array}$ & $\begin{array}{c}\text { Content of } \\
\text { CNT } \\
(\mathrm{Wt} \%)\end{array}$ & WCA $\left({ }^{\circ}\right)$ & SA1 $\left(^{\circ}\right)$ & SA2 $\left(^{\circ}\right)$ \\
\hline 1 & $10: 0$ & 0 & 105 & $>90$ & $>90$ \\
2 & $9: 1$ & 10 & 122 & $>90$ & $>90$ \\
3 & $4: 1$ & 20 & 131 & $>90$ & $>90$ \\
4 & $3: 1$ & 25 & 133 & $>90$ & 55 \\
5 & $2: 1$ & 33.33 & 150 & 18 & $<10$ \\
6 & $1: 1$ & 50 & 152 & 10 & 4 \\
7 & $1: 2$ & 66.66 & 154 & 10 & $<3$ \\
\hline
\end{tabular}


Table: 2 WCA $\left({ }^{0}\right)$ after heat treatment at different temperatures

\begin{tabular}{cccccccccc}
\hline PVDF: & Content of CNT & \multicolumn{7}{c}{ WCA $\left(^{0}\right)$} \\
MWCNT & $(\mathrm{Wt} \%)$ & $300 \mathrm{~K}$ & $373 \mathrm{~K}$ & $423 \mathrm{~K}$ & $473 \mathrm{~K}$ & $523 \mathrm{~K}$ & $573 \mathrm{~K}$ & $623 \mathrm{~K}$ \\
\hline $9: 1$ & 10 & 122 & 124 & 118 & 87 & 79 & 77 & $<10$ \\
$4: 1$ & 20 & 131 & 132 & 131 & 114 & 113 & 122 & $<10$ \\
$3: 1$ & 25 & 133 & 135 & 133 & 116 & 114 & 127 & $<10$ \\
$2: 1$ & 33.33 & 150 & 151 & 153 & 153 & 150 & 147 & $<10$ \\
$1: 1$ & 50 & 152 & 152 & 153 & 153 & 152 & 151 & $<10$ \\
$1: 2$ & 66.66 & 154 & 154 & 153 & 153 & 153 & 152 & $<10$ \\
\hline
\end{tabular}



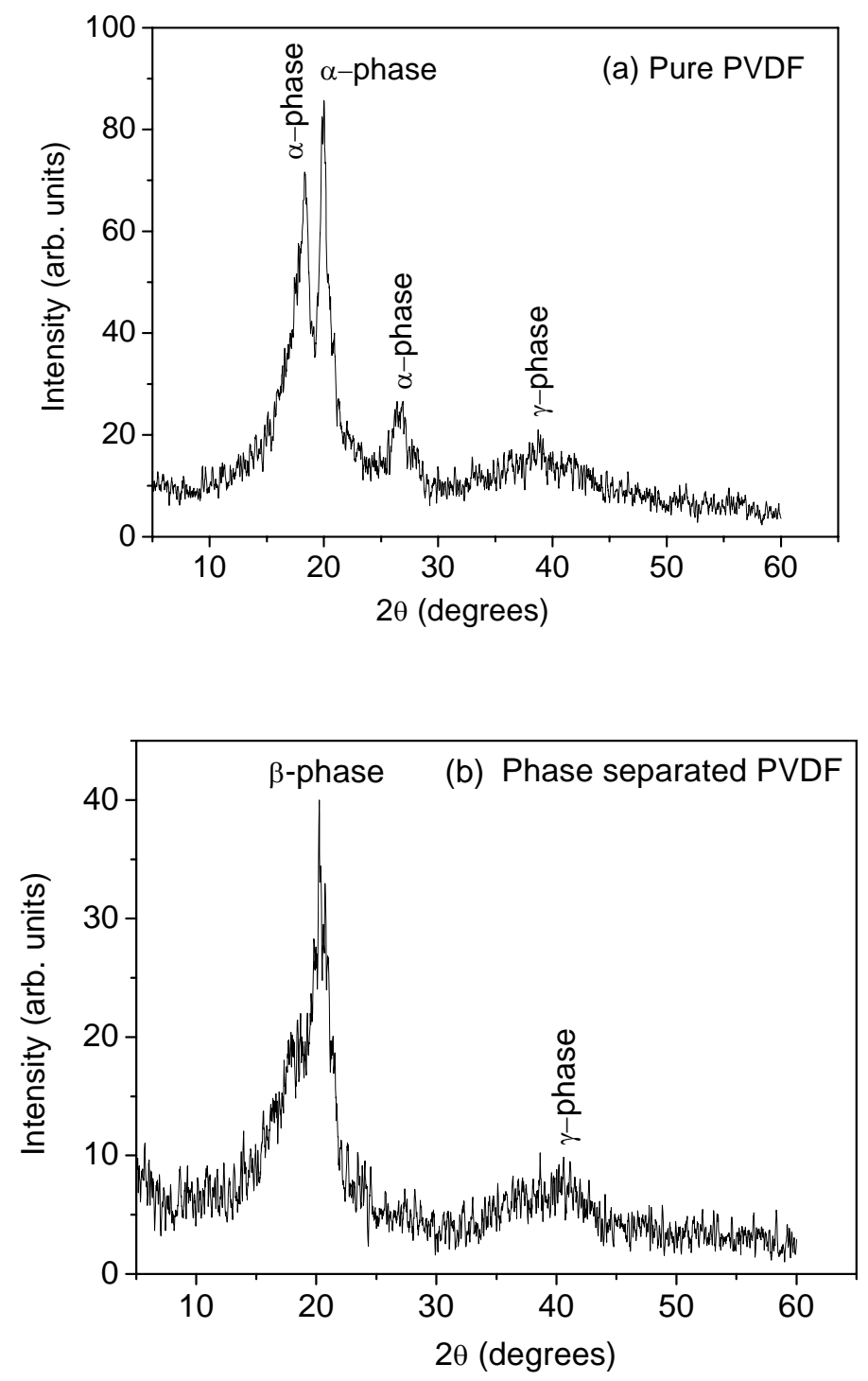

Fig. 1 X-ray diffraction pattern of (a) as-received PVDF and (b) phase separated PVDF. 

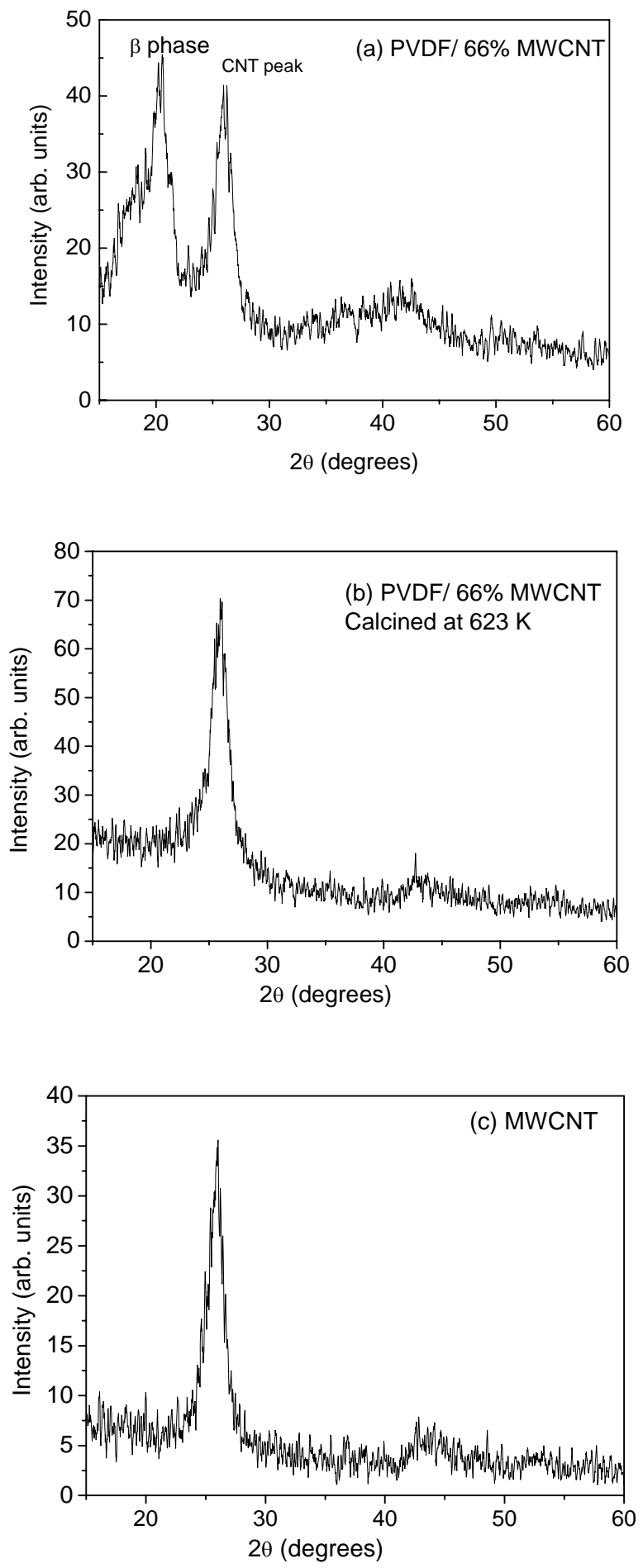

Fig. 2. X-ray diffraction pattern of PVDF-66 wt.\% MWCNT superhydrophobic coatings at (a) room temperature $(300 \mathrm{~K})$, (b) calcined at $623 \mathrm{~K}(1 \mathrm{~h})$ and (c) pure MWCNT 


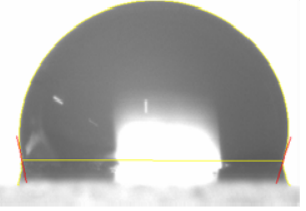

(a)

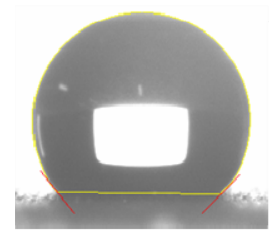

(d)

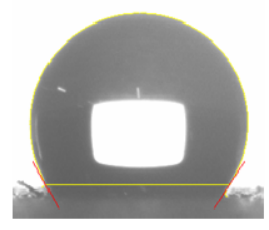

(b)

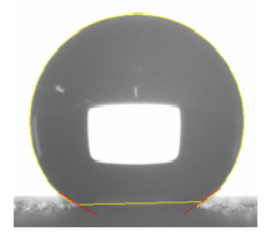

(e)

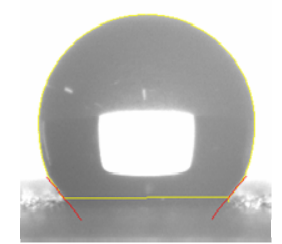

(c)

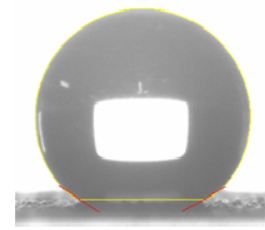

(f)

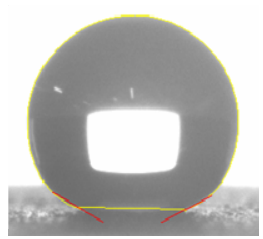

(g)

Fig. 3. Images of water drops on PVDF-MWCNT with different MWCNT concentrations: (a) $0 \%$, (b) $10 \%$, (c) $20 \%$, (d) 25\%, (e) $33.33 \%$, (f) $50 \%$, (g) $66.66 \%$. 


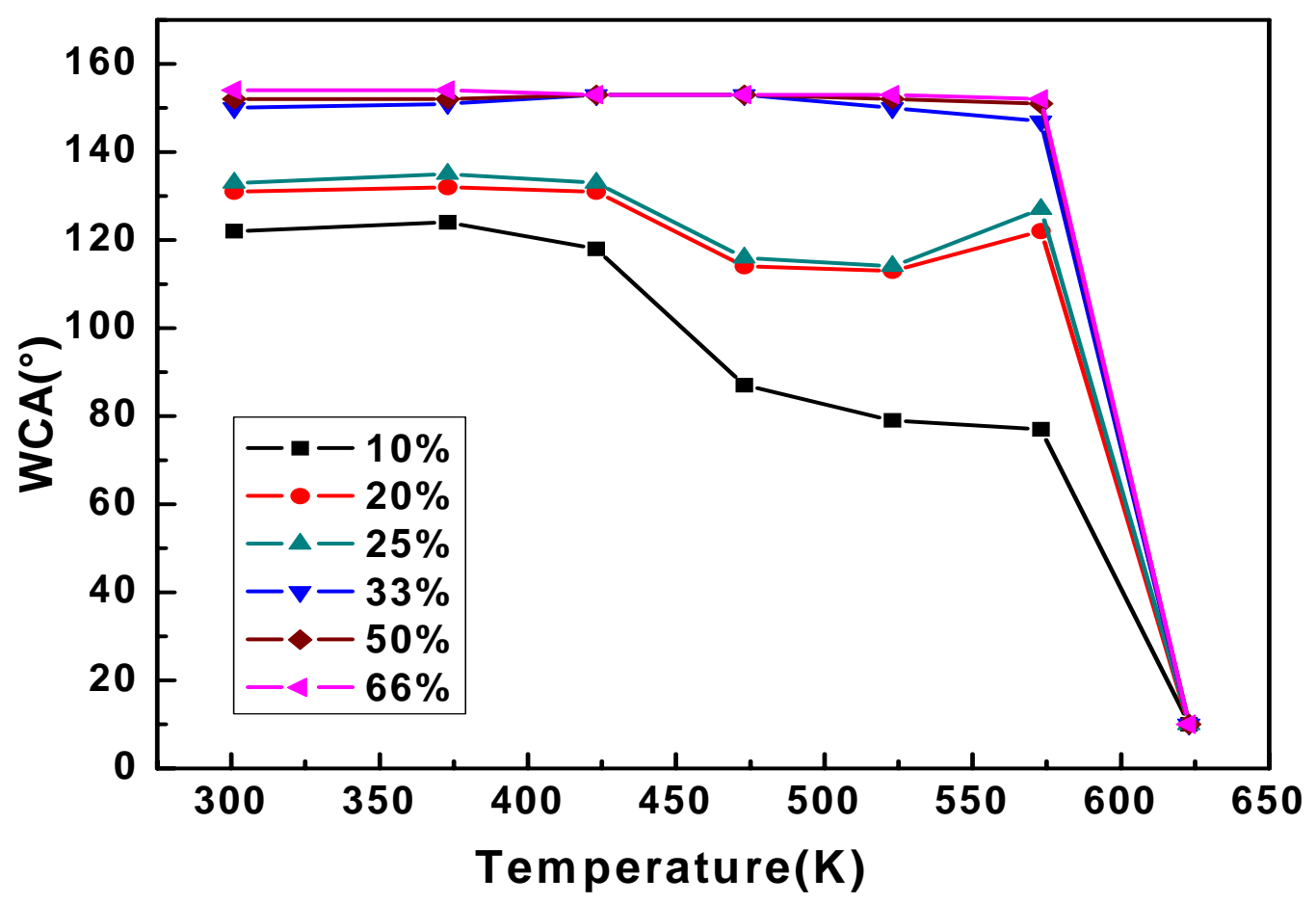

Fig. 4. Variation of WCA with different annealing temperature in PVDF/66\%MWCNT coatings 


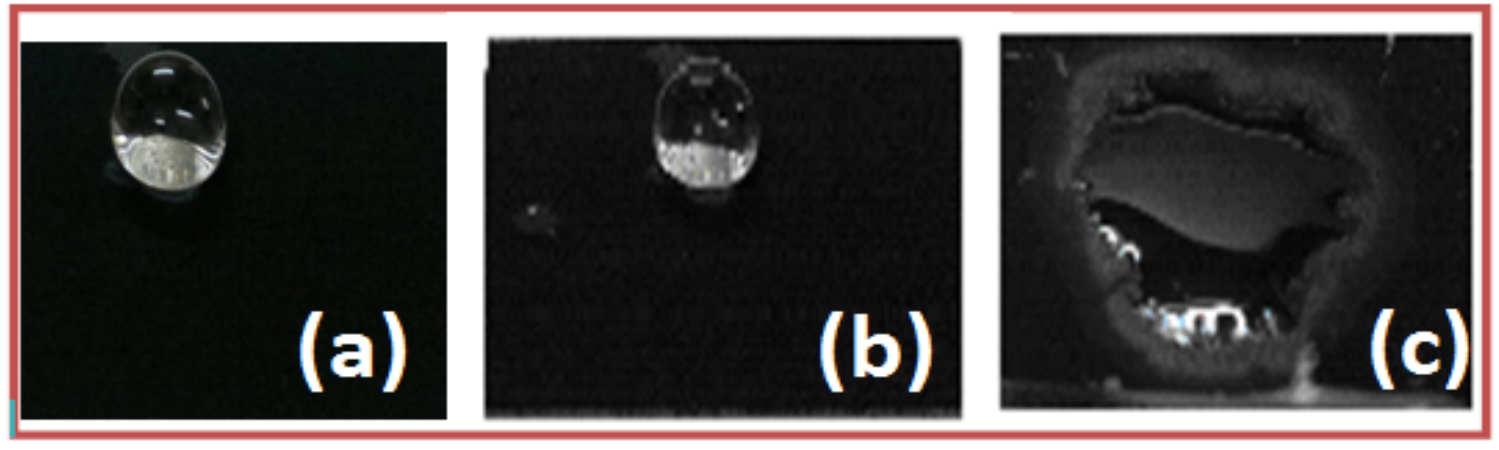

Fig. 5. Water droplets on the PVDF-MWCNT superhydrophobic coatings (a) $300 \mathrm{~K}$ and after heat treatment at (b) $573 \mathrm{~K}$ and (c) $623 \mathrm{~K}$ 


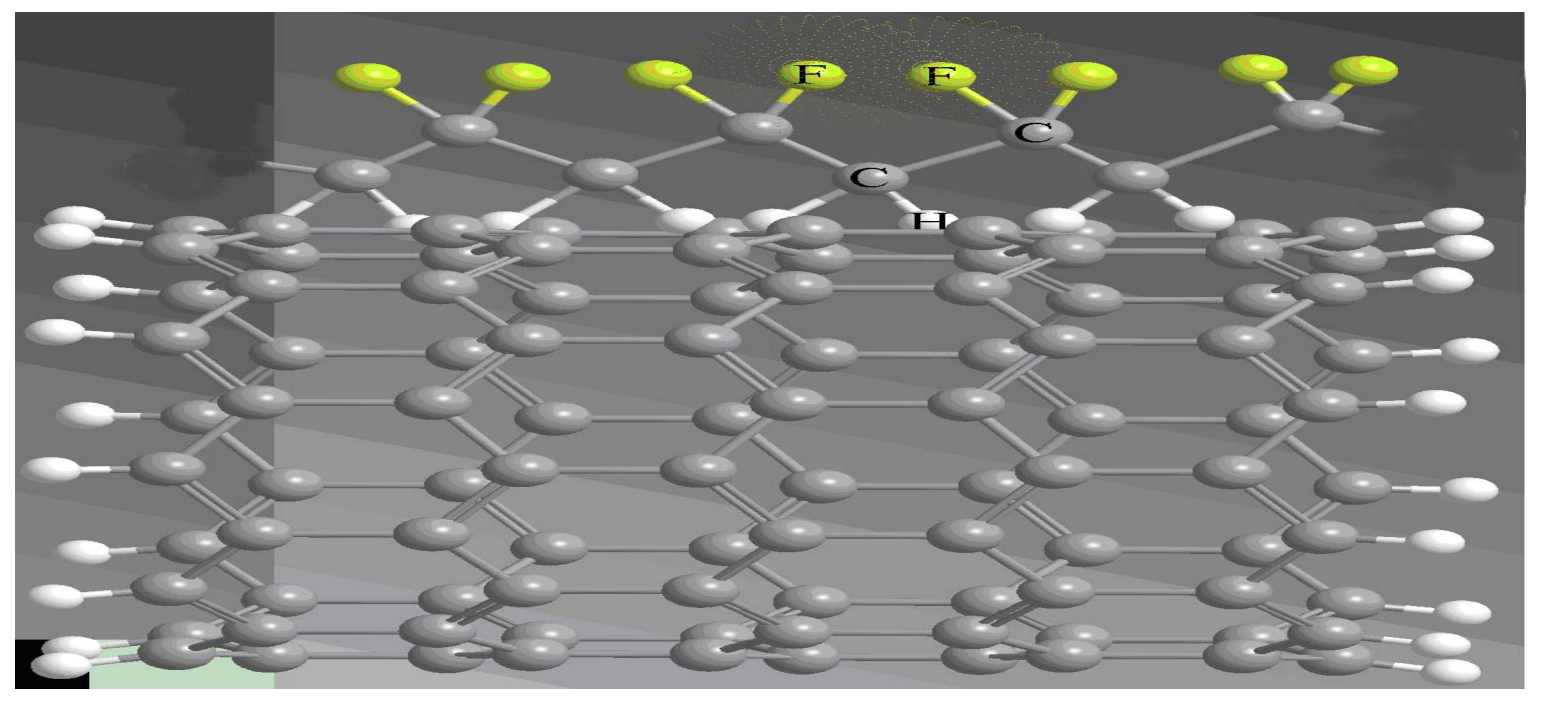

Fig. 6. Schematic representation of $-\mathrm{CF}_{2}-$ projection on the outer surface of MWCNT 

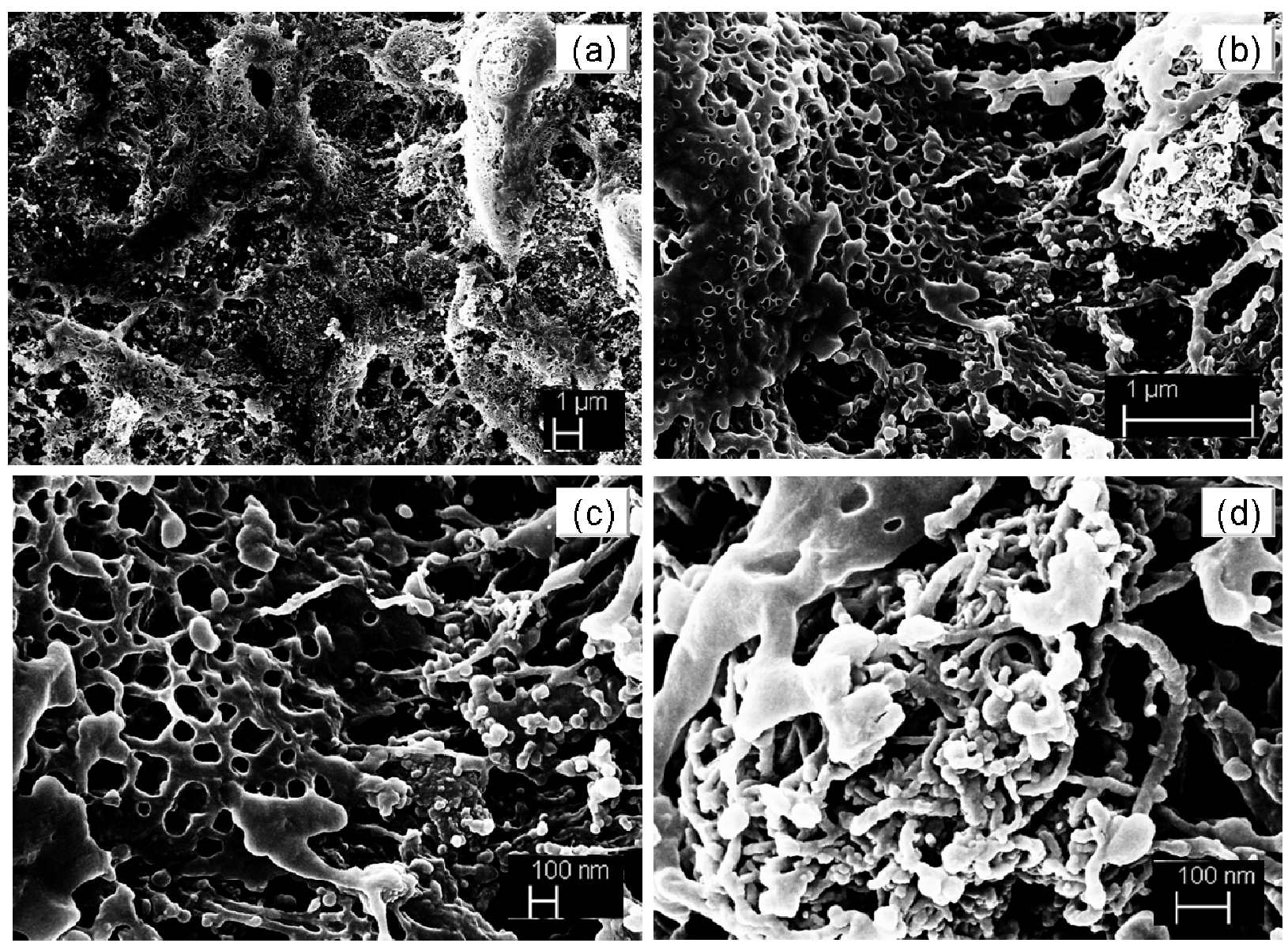

Fig. 7. FESM images of PVDF-MWCNT 10\% at different magnifications (a) 5KX, (b) $25 \mathrm{KX}$, (c) $50 \mathrm{KX}$, (d) $100 \mathrm{KX}$ 

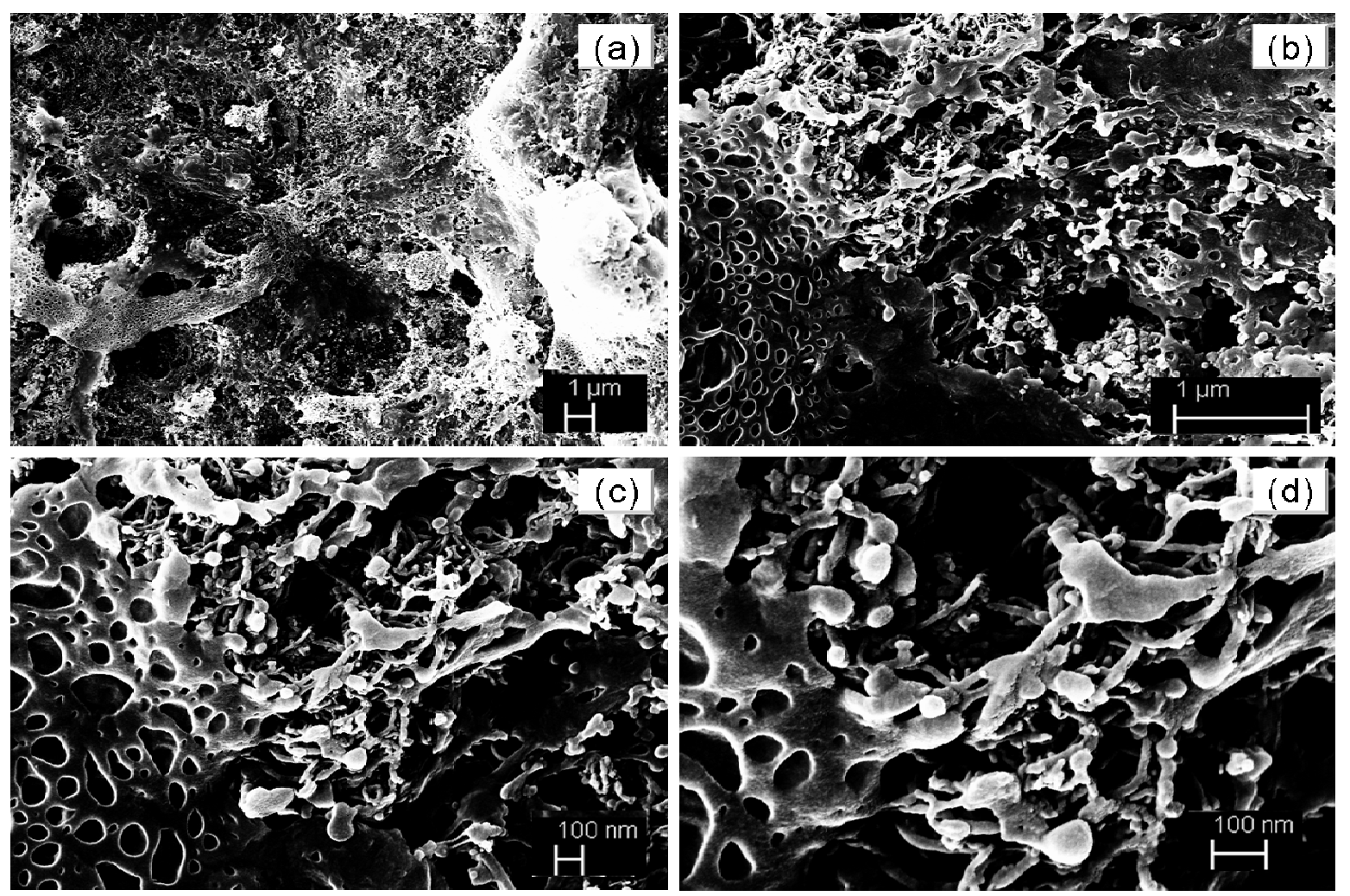

Fig. 8. FESM images of PVDF-MWCNT 33\% at different magnifications (a) 5KX, (b) $25 \mathrm{KX}$, (c) $50 \mathrm{KX},(\mathrm{d}) 100 \mathrm{KX}$ 

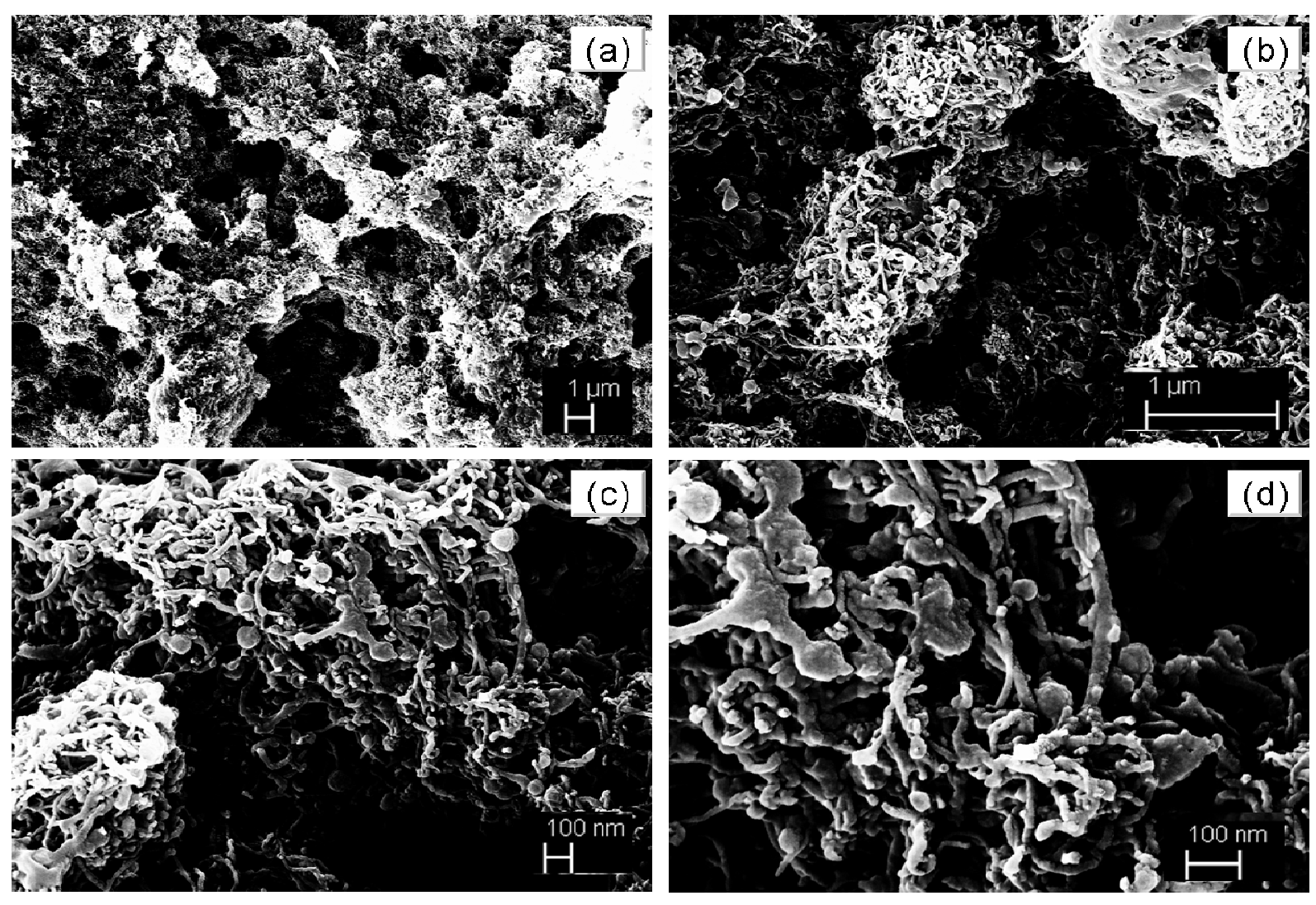

Fig. 9. FESM images of PVDF-MWCNT 66\% @ RT for different magnifications
(a) $5 \mathrm{KX}$
(b) $25 \mathrm{KX}$,
(c) $50 \mathrm{KX}$,
(d) $100 \mathrm{KX}$ 


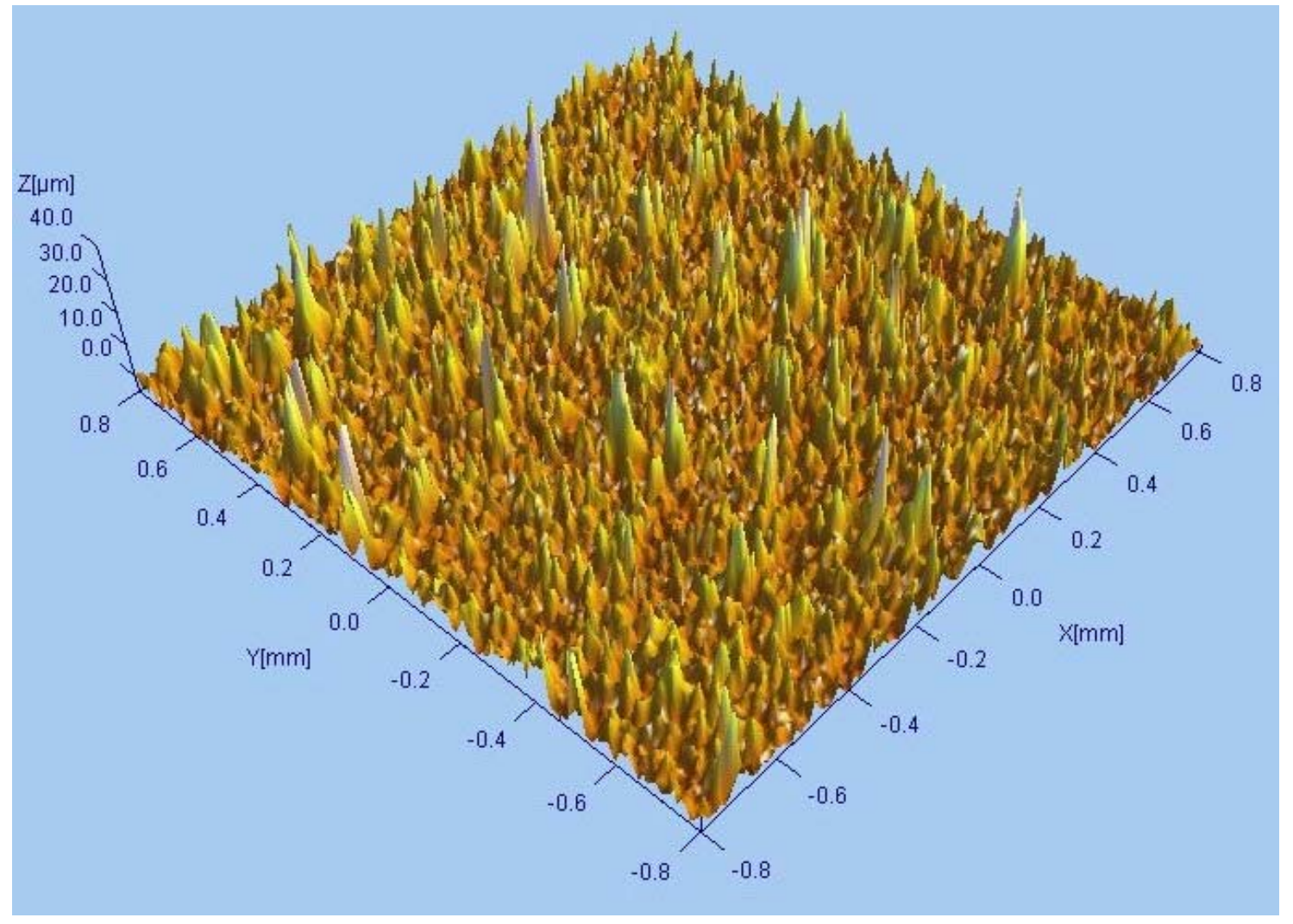

Fig. 10. 3D surface roughness of $66 \%$ MWCNT-PVDF superhydrophobic coating 

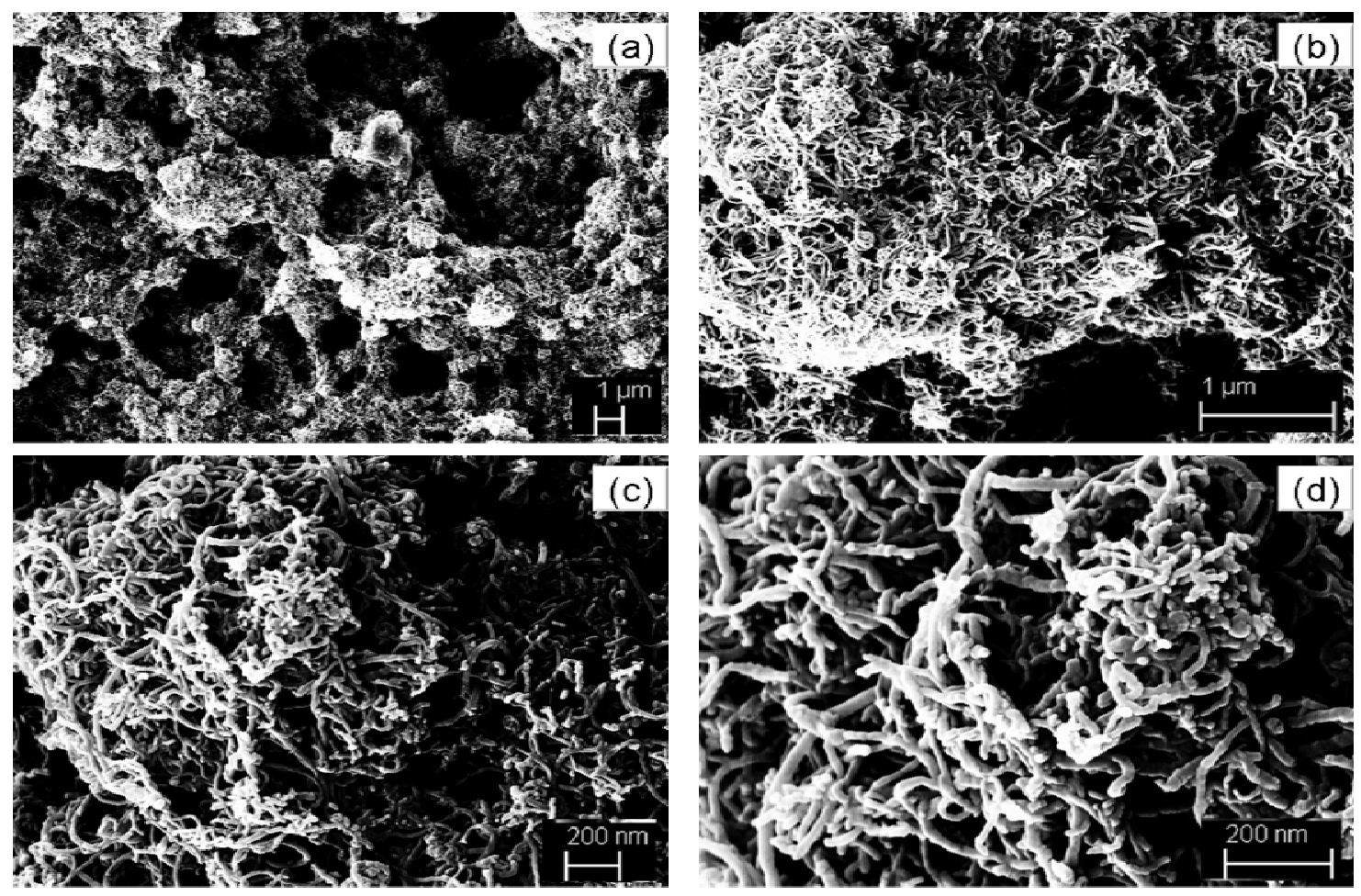

Fig. 11. FESM images of PVDF-MWCNT 66\% after heating at $623 \mathrm{~K}$ at different magnifications (a) $5 \mathrm{KX}$, (b) $25 \mathrm{KX}$, (c) $50 \mathrm{KX}$, (d) $100 \mathrm{KX}$ 
(a) $33 \%$ RT

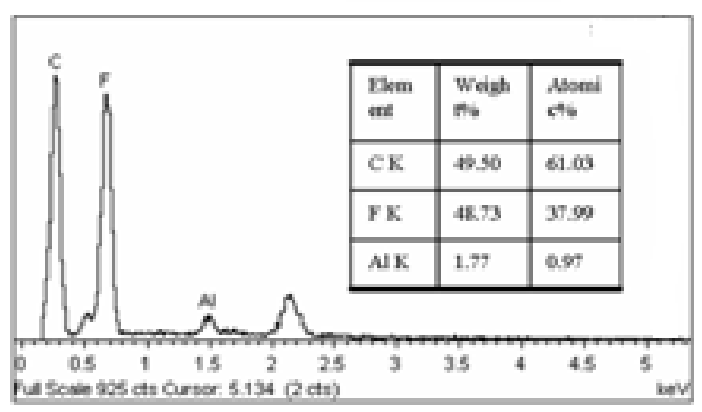

(b) $66 \%$ RT

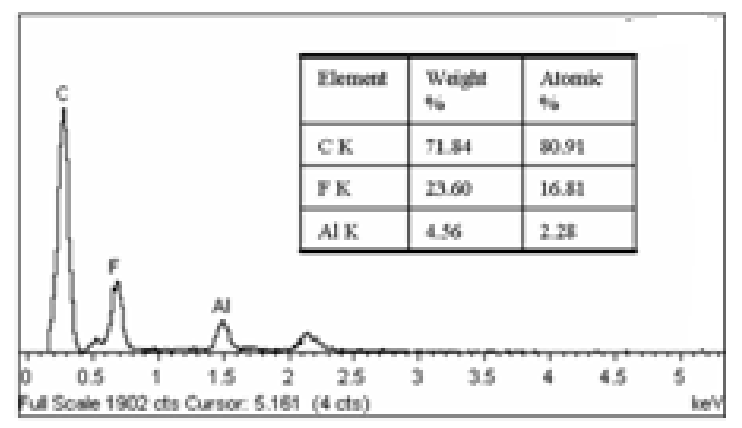

(c) $66 \% 623 \mathrm{~K}$

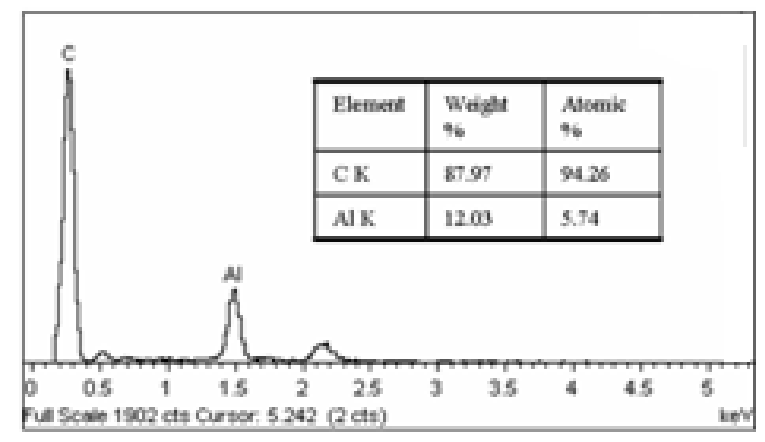

Fig. 12. Energy dispersive X-ray spectra of (a) 33\% (b) $66 \%$ and (c) $66 \%$ calcined at 623K PVDF-MWCNT coatings 

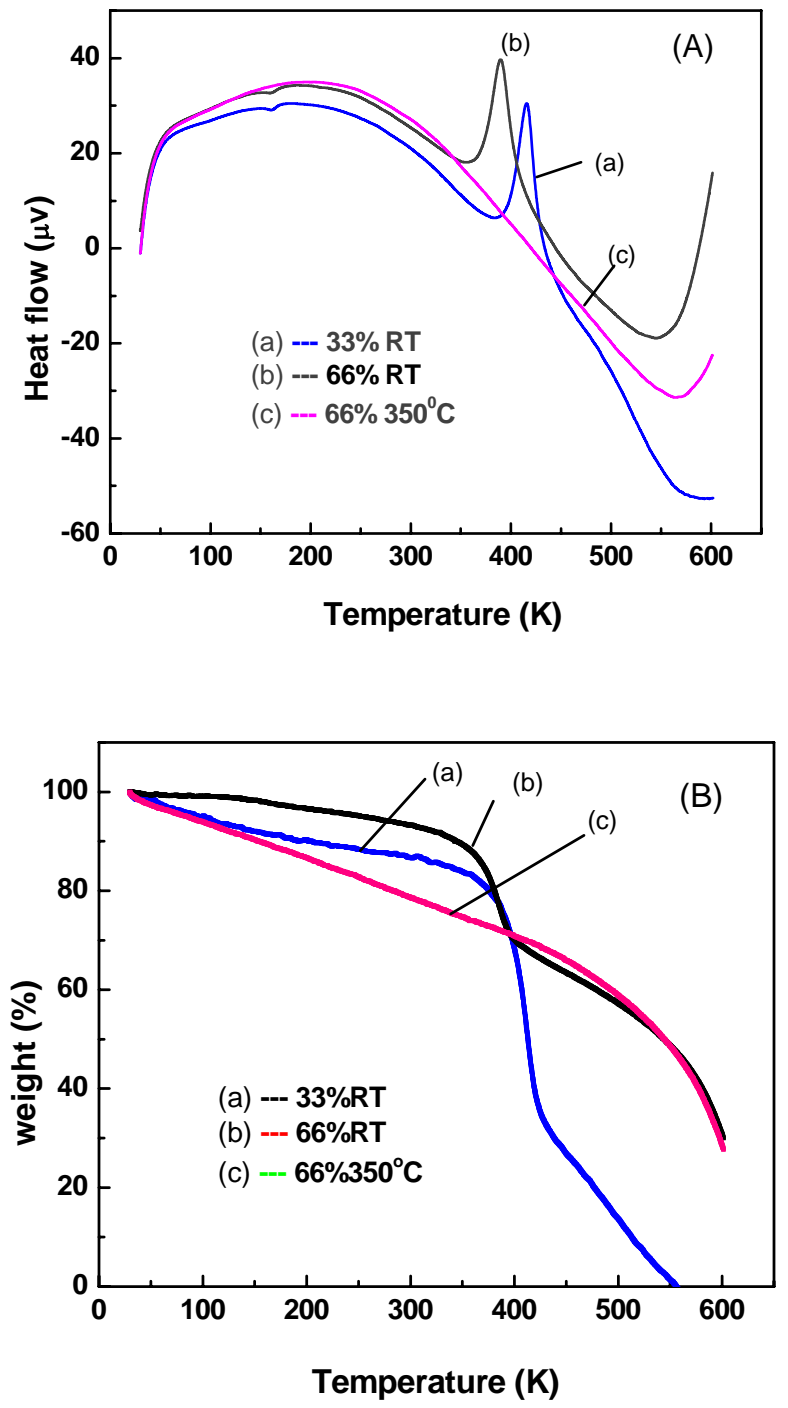

Fig. 13. (A) DTA curves and (B) corresponding TGA curves of PVDF-MWCNT with different MWCNT concentrations: (a) 33\%, (b) 66\%, and (c) 66\% calcined at $623 \mathrm{~K}$ 


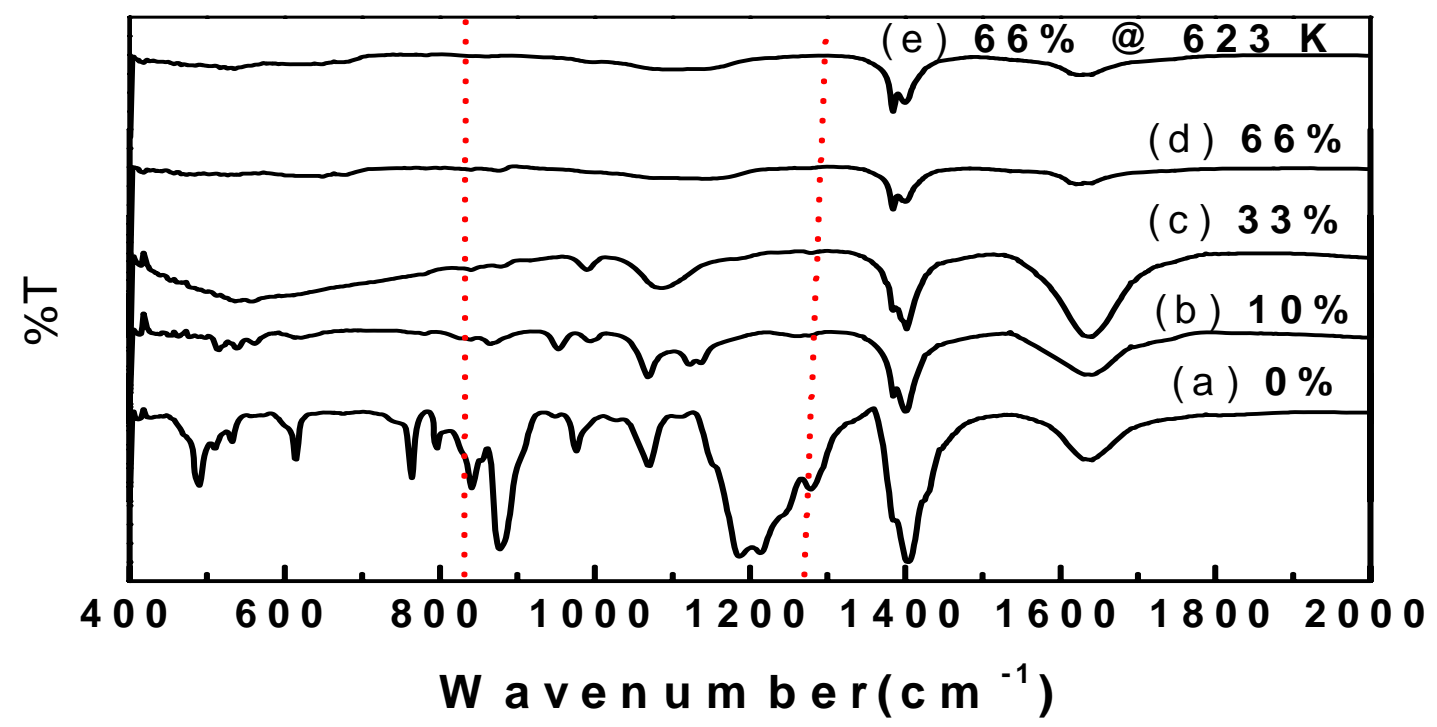

Fig. 14. FTIR spectra for PVDF-MWCNT with different MWCNT concentrations:

(a) $0 \%$, (b) $10 \%$, (c) $33 \%$, (d) $66 \%$, and (e) $66 \%$ claimed at $623 \mathrm{~K}$ 


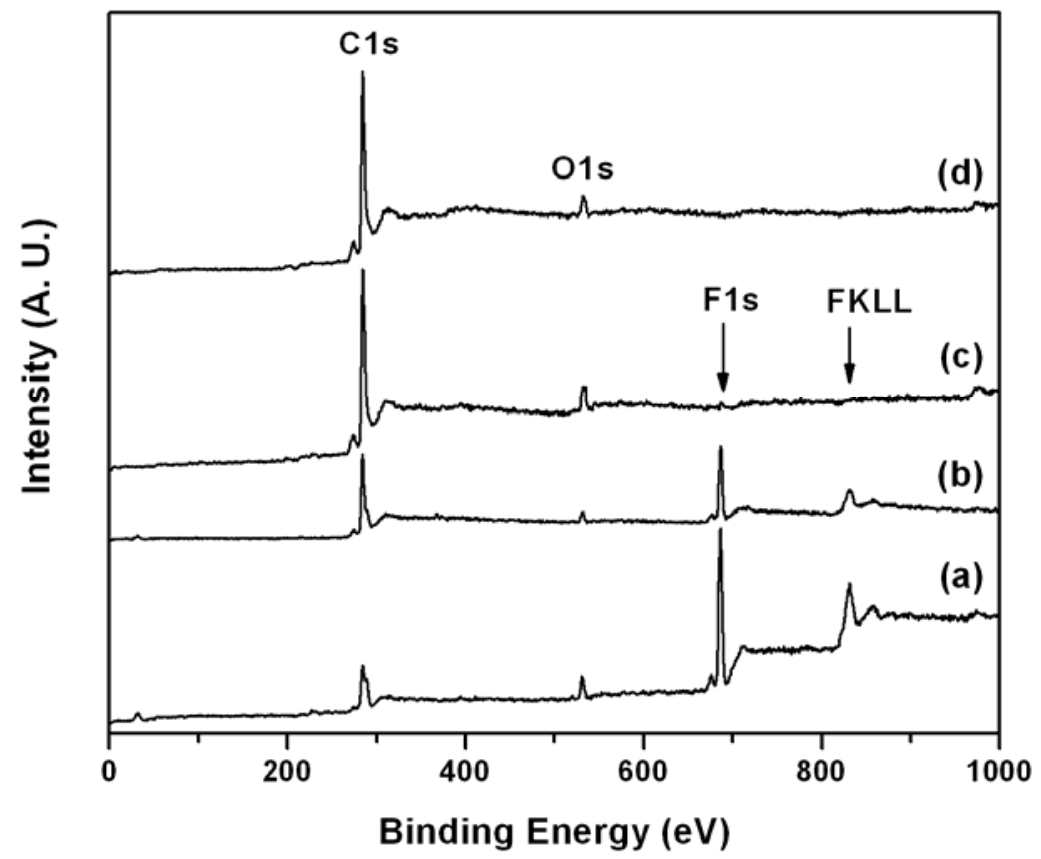

Fig. 15. Survey spectra of (a) pure PVDF, (b) PVDF-66\% MWCNT, (c) PVDF-66\% MWCNT after heated at $350{ }^{\circ} \mathrm{C}$ and (d) pure MWCNT. 


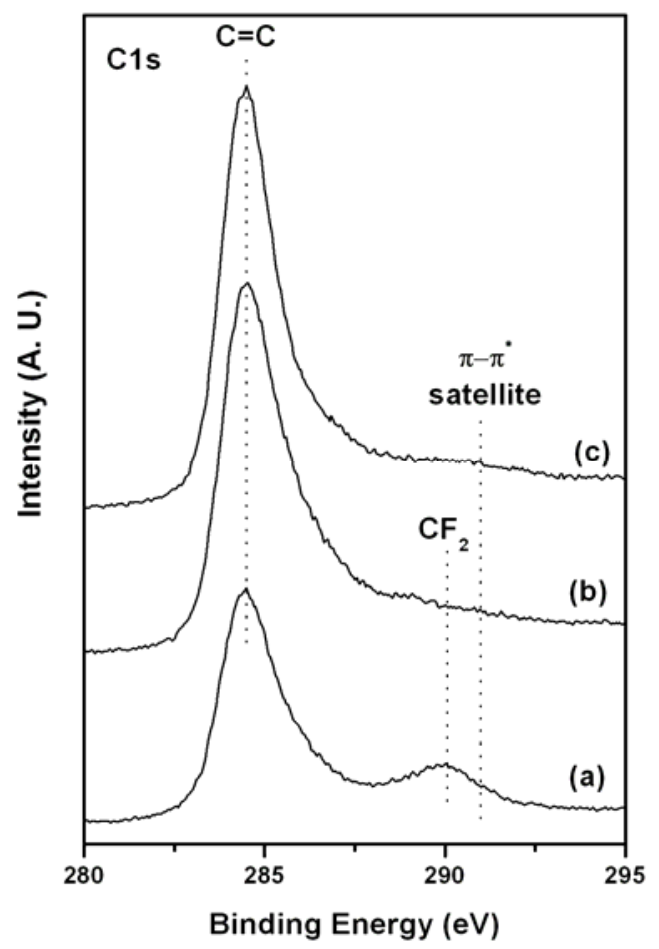

Fig. 16. C1s core level spectra of (a) as-prepared PVDF-66\% MWCNT and (b) PVDF-66\% MWCNT heat treated at $350{ }^{\circ} \mathrm{C}$ and (c) pure MWCNT. 


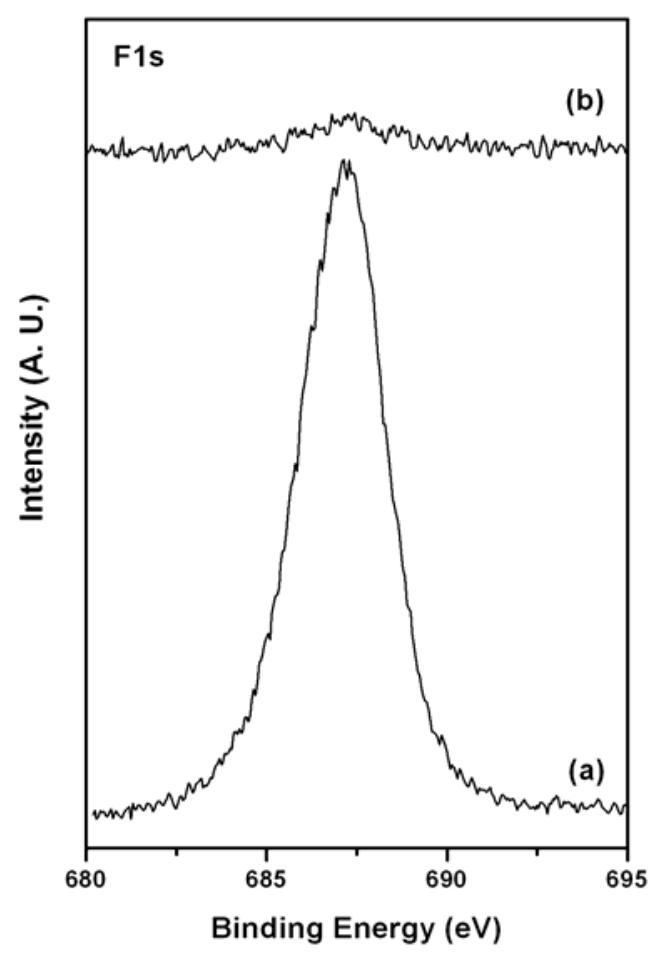

Fig. 17. F1s core level spectra of (a) as-prepared PVDF-66\% MWCNT and (b) PVDF-66\% MWCNT heat treated at $350{ }^{\circ} \mathrm{C}$. 